Nathan Wachtel. Histoire et anthropologie

\title{
Sous le regard de l'ancêtre : biographie et autobiographie en anthropologie
}

Carlo Severi

\section{(2) OpenEdition}

\section{Journals}

Édition électronique

URL : http://journals.openedition.org/actesbranly/720

ISSN : 2105-2735

\section{Éditeur}

Musée du quai Branly Jacques Chirac

Référence électronique

Carlo Severi, « Sous le regard de l'ancêtre : biographie et autobiographie en anthropologie », Les actes de colloques du musée du quai Branly Jacques Chirac [En ligne], 7 | 2016, mis en ligne le 15 novembre 2016, consulté le 08 septembre 2020. URL : http://journals.openedition.org/actesbranly/720

Ce document a été généré automatiquement le 8 septembre 2020

(c) Tous droits réservés 


\title{
Sous le regard de l'ancêtre : biographie et autobiographie en anthropologie
}

\author{
Carlo Severi
}

\section{Carlo SEVERI}

Merci de m'avoir invité pour cet hommage à Nathan auquel je participe avec grand plaisir. Je me sens honoré de parler en m'appuyant sur son travail. Je lui dois beaucoup. J'ai été comme pratiquement tous les ethnologues de ma génération impressionnée par La vision des vaincus. Et comme nous tous, $\mathrm{j}$ 'ai admiré Le retour des ancêtres.

2 Mais l'ouvrage m'ayant particulièrement touché reste La foi du souvenir constituant une sorte de labyrinthe de vie et de témoignages. J'ai été très impressionné par la vie que ces personnes acquièrent au cours de la lecture. Ce sont souvent des vies perdues et gâchées. Cela est longtemps resté dans ma mémoire au point que, pas plus tard qu'il y a trois à quatre semaines, je citais le cas de ce prisonnier juif à Mexico au XVI ${ }^{\mathrm{e}}$ siècle communiquant avec les autres prisonniers en Nahuatl.

D'ailleurs, j'en parlais avec Federico NAVARRETE lequel m'a raconté que le Nahuatl était la langue des marchands à Mexico. Derrière cette trace, il existe toute une mesure qui, selon moi, durera très longtemps dans notre réflexion d'anthropologue.

En effet, je me suis également intéressé au problème de la biographie et de la mise en place d'informations biographiques en anthropologie. Je souhaiterais présenter quelques remarques ouvertes sans beaucoup de réponses, mais avec des questionnements sur la question du terrain anthropologique. En réalité, mon premier réflexe sur le terrain a été de rencontrer des thérapeutes «Kuna » et de leur demander de raconter leur vie.

D'ailleurs, j'avais récolté plusieurs autobiographies effectuées devant l'ethnologue. Cela prenait en compte l'ombre de l'observateur. J'avais effectué une sorte de critique et discussion sur deux types autobiographiques «Kuna»: le "Kuna Andorre », le «Kuna héroïque », le «Kuna» se dressant contre les Blancs, le «Kuna» faisant l'éloge de la tradition et montrant son savoir. Je l'ai appelé « José », mais il s'appelait autrement. Un 
autre personnage parlait plutôt de ses passions et de la terreur que les démons de la folie pouvaient faire circuler dans un village. Ils parlaient beaucoup plus directement d'argent.

6 En effet, c'est avec le second que j'ai pu travailler et non avec le premier. Cette affaire m'avait permis de prendre conscience d'une sorte de présence du genre biographique à l'intérieur du terrain. Comme premier réflexe, il était probablement nécessaire d'analyser le rôle qu'ont joué la biographie et l'autobiographie et cette expérience de terrain dans l'histoire de l'anthropologie. Il s'agissait de la première partie.

7 À l'époque, j'avais publié un papier dans Quaderni Storici. Il me semble que Giovanni LÉVI et Carlos GINZBURG étaient présents. Nous nous étions retrouvés à Bologne avec des historiens. Il s'agissait d'un épisode de discussion entre anthropologues et historiens sur la clé narrative que le thème biographique pouvait présenter. J'aborderai brièvement le rôle de la biographie à l'intérieur de l'histoire de l'anthropologie pour ensuite poser un problème et proposer une solution ouverte à la discussion.

8 En me penchant sur l'anthropologie fondée sur le récit autobiographie concernant des classiques tels que Black Elk Speaks, la première chose qui m'avait intéressé était que dans le cours du récit le lecteur se familiarise peu à peu avec celui ou celle dont est contée l'histoire jusqu'à avoir une sorte d'illusion de partager le même monde culturel. Toute distance semble s'abolir et l'horizon à l'intérieur duquel se déplace le narrateur, même s'il est constitué de rites étranges et de croyances inattendues, se teinte de la couleur du vraisemblable. Cette sensation semble simplement le reflet d'une trop évidente situation d'énonciation. Un homme ou une femme généralement âgés confient l'histoire de leur vie à une tierce personne. Il s'agit de l'un des scénarios de l'anthropologie.

9 Des années plus tard, un lecteur ignorant de l'histoire et de la culture du narrateur se projette dans son récit et croit le comprendre sans nulle intermédiation. Nous nous sentons frères. Or cette apparente immédiateté dissimule une situation discursive autrement complexe et ambiguë dont je souhaiterais éclairer quelques aspects. La sensation de vraisemblance et de familiarité de la part du lecteur est précisément liée à l'entrelacement de matériaux hétérogènes tissant la trame narrative du récit biographique. Cette espèce particulière d'ambiguïté est sans doute le trait le plus saillant de la biographie du moins telle que l'ont pratiquée les anthropologues et non pas Nathan qui me semble une grande exception dans cette affaire. Qu'est-ce nous retrouvons derrière cette efficacité et cette vraisemblance? Quel est le dispositif d'énonciation en rendant compte en réalité?

10 Tout d'abord, ce type de biographie est très rare dans les sociétés amérindiennes comme genre de la littérature orale. Nous ne retrouvons pas une autobiographie sensible et remplie d'épisodes dans le monde des Indiens des Plaines qui est d'ailleurs un des lieux classiques de ce scénario anthropologique. En fait, nous retrouvons une autobiographie chantée sélectionnant rigoureusement les éléments représentés en pictographie. Il s'agit de guerre ou de chasse. Nous trouvons également quelques pictogrammes concernant la cour faite aux femmes, mais cela reste mineur dans cette littérature pictographique sur laquelle j'ai pu travailler.

11 Par ailleurs, ce genre littéraire pictographique ne ressemble presque en rien aux biographies que nous retrouvons dans la littérature anthropologique. Autrement dit, nous avons rarement des témoignages, par exemple, de mise en place de sensation et d'impression d'enfance. Les Indiens se taisent sur ces éléments. Nous pourrions dire la 
même chose de l'Amazonie. D'ailleurs, un magnifique ouvrage de Suzanne OAKDALE concerne les Cayabi où nous retrouvons un genre ritualisé d'autobiographie chez les chamanes Cayabi très impressionnant du point de vue européen.

En effet, ces stéréotypes se répètent et se succèdent. Par contraste, j'ai un peu regardé dans la littérature anthropologique. Si la biographie n'est pas un genre des littératures orales amérindiennes, cela est par contre un genre ayant connu un succès incroyable en anthropologie. Il existerait cinq cent soixante-dix-sept biographies ou autobiographies concernant les Indiens des plaines et les Inuits. Cela a constitué un succès important dans la manière de rendre compte de ces cultures. Si cela n'est pas un genre littéraire de littératures orales amérindiennes, il s'agit certainement d'un genre littéraire faisant partie de la littérature qu'est l'anthropologie.

13 Toutes ces biographies ont toujours deux auteurs, c'est-à-dire la personne qui s'assied et qui raconte son histoire et celui ou celle avec son magnétophone qui coupe, corrige, reprend et rend le récit plus rapide ou plus long. Nous avons affaire à une sorte de rencontre littéraire entre anthropologues et le récit de son interlocuteur.

14 À partir de ce moment, l'anthropologie semble réagir à cette situation presque de "prison-miroir » et de mise en abyme entre l'auteur et celui faisant le récit d'une manière tout à fait précieuse, mais des problèmes subsistent. La réaction est de déplacer le foyer de l'attention de l'interlocuteur à l'anthropologue lui-même. Il existe deux grands ouvrages: Journal d'ethnographe de MALINOWSKI et Tristes Tropiques de LÉVI-STRAUSS qui sont deux ouvrages différents. En effet, Tristes Tropiques est un chefd'œuvre littéraire tandis que le journal de MALINOWSKI a été publié sous le titre A diary in the strict sense of the term. Nous voyons cet homme se lever le matin désespéré d'avoir mal dormi et il prend des drogues le soir. L'ethnographie qu'il tente de faire représente une bataille quotidienne. Nous remarquons le désespoir de la personne. Dans Tristes Tropiques, nous entrons dans un chef-d'œuvre dela pensée du XXe siècle. Cela est tout à fait autre chose bien que LÉVI-STRAUSS indique avoir écrit dans un état perpétuel d'exaspération.

15 En même temps, cette sorte de polarité se met en scène dans le scénario anthropologique. D'abord, nous retrouvons l'apparente présence directe du narrateur. Puis, celui qui écoute commence à écrire et à exprimer ses propres pensées. Il s'agit d'un autre sujet apparaissant sur scène. Mais, je me suis demandé si nous pouvions sortir de ce cercle. Pouvons-nous faire de l'anthropologie des biographies telles qu'elles apparaissent stéréotypées et répétitives ou moins compréhensives que ces situations directes telles que nous les voyons apparaître dans le monde amérindien? Je crois que cela est possible, à condition d'étudier les formes de fabrication d'une mémoire même biographique et autobiographique. Je souhaiterais en donner un exemple.

16 Pour respecter cette dialectique entre l'auteur et la tradition étudiée, je prendrai la tradition des Apaches de l'Ouest. Un formidable linguiste Keith BASSO s'est occupé de cette question de la biographie. Je n'aurais probablement pas le temps de parler de l'auteur qui est un singulier personnage. En même temps, ce dernier partage avec Nathan une attention à l'image inversée du Blanc dans la culture indienne.

D'ailleurs, dans l'ouvrage fantastique Portraits de l'homme blanc, il nous fait part de ce genre littéraire oral pratiqué par les Apaches de l'Ouest consistant à réaliser des portraits naturellement grinçants, ironiques, durs et très drôles de l'homme blanc vu par les Apaches. Mais, pour nous parler d'histoire de vie, BASSO prend un autre point de vue et travaille sur l'espace. Cela paraît tout de suite étonnant. Je crois qu'il convient 
de suivre un peu son raisonnement. BASSO a écrit un livre qui vient de sortir en français dont le titre est L'eau se mêle à la boue dans un bassin à ciel ouvert avec pour titre original Wisdom sits in places.

18 En effet, ce dernier articulera la notion de lieu avec celle de biographie. Avant tout, il s'agit de répondre à une commande du conseil tribal demandant à l'ethnologue de réaliser une carte géographique de la réserve. Ils avaient déjà les cartes qui étaient celles de l'armée. D'ailleurs, ils sont bien placés pour le savoir, parce que comme tous les pauvres aux États-Unis, les Apaches de l'Ouest travaillent souvent à l'armée. Ils demandent plutôt une carte avec les noms de lieux en apache. Puis, il va falloir voir comment sont faits ces noms et ce que nous pouvons en tirer pour la culture apache. Je ne vous présenterai pas la stratégie de BASSO sur cette affaire, mais le cas échographique qui nous intéresse.

En effet, BASSO commence par identifier dans le savoir géographique des Apaches le niveau le plus spécifique de l'espace physique, c'est-à-dire la désignation des lieux. Par exemple, un lieu dans lequel nous pouvons traverser un ruisseau s'appellera "eau s'écoule au-dessus d'une succession de rochers plats ». D'autres lieux peuvent s'appeler "groupes compacts de rochers blancs en hauteur » ou encore « eau s'écoule en dessous d'un peuplier ». Ces lieux renvoient évidemment à un ensemble d'histoires pour ne pas dire de mythes. Il existe évidemment des mythes dans ces histoires, mais il y a également ce qu'il s'est passé quand un jeune n'a pas respecté un interdit et s'est trouvé sous la vengeance, par exemple, d'un éclair. Ce dernier est mort, parce qu'un Gan (un des esprits des ancêtres) s'est vengé contre lui parce qu'il avait transgressé un interdit. Ce sont parfois des histoires d'amour.

Ces lieux sont donc liés à une mémoire. Mais l'originalité de BASSO est de trouver le terme intermédiaire. Autrement dit, c'est la «vis » reliant ces noms de lieux aux histoires ayant entraîné une espèce de littérature projetée dans un territoire. Il s'agit d'une sorte de pensée géographique incluant les histoires des gens et, avant tout, la présence des ancêtres. Quel est donc l'opérateur de cette inscription du lieu dans la mémoire sociale ? Comment le langage transcrit-il la présence de cet opérateur?

21 BASSO commence par montrer qu'un nom de lieu en apache est une «linguisticallyformulated image ». Il s'agit d'une image décrite par des mots. Ensuite, ce dernier démontre qu'un nom de lieu apache implique toujours une direction du regard. Tel qu'il est décrit par l'image verbale, ce lieu ne sera littéralement pas visible si nous ne prenons pas un point d'observation très précis. D'un tout autre point de vue, les pierres servant à nommer ce lieu ne se présenteront pas au-dessus d'une succession de rochers plats, par exemple, comme le veut la désignation verbale.

Dans le troisième cas cité, le peuplier et lui-même ne pourront pas fonctionner comme un point de repère dans la direction de l'eau (ce qui est crucial pour la désignation du lieu) si nous ne le percevons pas selon une certaine direction du regard. Pour que cette géographie reçoive une cohérence, il convient toujours d'identifier un point dans l'espace impliquant la présence d'un observateur. Or cet observateur est l'ancêtre.

23 À partir de ce point de la description verbale, pour acquérir un sens et servir à désigner un lieu, il doit être énoncé. N'importe quelle personne passant par là pourra naturellement occuper ce point d'observation. Mais, pour les Apaches, ce lieu indique également la place de l'ancêtre qui, occupant pour la première fois ce point dans l'espace, a ainsi vu le lieu et l'a nommé tel qu'il le voyait. L'ancêtre n'a donc pas seulement marqué ce lieu d'un nom propre pour le distinguer d'autres lieux. Il a 
également inscrit sa propre présence invisible dans la description verbale du lieu, puisqu'il y a transcrit une trace de son regard.

Toute personne passant par cet endroit se met donc à la place de l'ancêtre. La parole qu'il ou elle énonce, le nom du lieu est celui de l'ancêtre. Celui qui voit et qui énonce le lieu voit donc le lieu à partir de ses yeux. La tâche qui lui a été confiée par le président du conseil de la tribu de reprendre une carte du point de vue des Apaches conduit BASSO à une découverte.

En effet, certains lieux sont pour les Indiens des lieux d'identification rituelle avec l'ancêtre. Un ruisseau, un arbre ou une pierre blanche marque la présence de l'ancêtre fondateur pouvant être humain ou animal (souvent animal) dans l'acte même d'adresser le regard. Pour ressentir cela, il suffit de voir les lieux du point de vue qui est le leur. Un paysage ne se réduit donc pas à une liste de noms de lieux que nous énumérons. BASSO ne décrit pas des itinéraires et n'énumère pas seulement des noms de lieux, mais il fait surgir un regard, celui des Apaches où l'espace prend la place du temps. Qu'est-ce que cela nous apprend? Cela nous apprend que la géographique fonctionne comme un ensemble d'images lesquels se lient toujours à des opérations de narration.

BASSO a également étudié un genre littéraire apache dénommé Speaking with names (parler avec des noms propres). Dans la conversation, le silence est toujours important. Pour commenter une histoire, nous évoquons une histoire par le nom du lieu. Nous parlons donc de « rocher plat et blanc » et nous offrons ainsi à l'imagination de celui ou de celle qui écoute de retrouver une histoire, puisqu'elle est ancrée dans tel ou tel lieu. Nous avons une sorte de clé géographique pour la narration des histoires de vicissitudes quotidiennes des gens et également des mythes.

Mais, tout cela se passe sous le regard de l'ancêtre. Il y a un autre interlocuteur, un autre témoin, car l'ancêtre est présent. En effet, nous sommes dans un lieu organisé par son regard. Nous pourrions nous arrêter à ce point, mais comme vous le savez sans doute, je cours depuis une vingtaine d'années après une théorie générale des modalités de la mémoire indigène a minima dans le monde amérindien. Cette séquence d'images véhiculée par des mots s'inscrit dans l'univers des arts de la mémoire amérindienne. D’ailleurs, j'avais évoqué cet aspect avec Nathan. Ce dernier m'avait indiqué que le papier était plus ou moins lisible, mais qu'il était nécessaire de réaliser un ouvrage. Je n'ai peut-être pas réalisé cet ouvrage, mais j'ai tout de même un livre en épreuve où nous parlons de ces éléments que j'enverrai prochainement à Nathan.

De ce point de vue, cela est intéressant, parce que nous retrouvons finalement ce que nous avons dans les arts de la mémoire classique. Autrement dit, nous retrouvons une iconographie des noms de lieux et des histoires liées à cette iconographie presque de la même manière que dans un champ rituel, en trouvant le lien avec des problèmes de narration et d'itinéraires. Mais, en même temps, les iconographies sont reconnues et non produites. Par ailleurs, les Apaches disposent d'une pictographie intéressante.

29 Toutefois, nous avons une sorte de fonctionnement pictographique confié à la formulation linguistique d'une image qui est la clé pour la mémoire de la vie des genres, aussi bien pour la biographie que pour l'autobiographie. Nous avons probablement une manière de renouveler ce champ et ce scénario traditionnel de la narration pseudo autobiographique ou pseudo biographique et de sortir de cette sorte de mise en abyme de l'interlocuteur indigène et de l'énonciateur anthropologue à 
travers l'étude des formes et non pas des contenus de distribution de ce savoir, de cette littérature et même de l'expérience biographique. Merci. (Applaudissements).

30 Mme Carmen BERNAND

Nous donnons la parole au linguiste anthropologue William HANKS.

\section{RÉSUMÉS}

Dans cette communication, je vais comparer la manière d'appréhender le conflit avec les Blancs qui caractérise deux traditions chamaniques amérindiennes: les Kuna de San Blas (Panama) et les Apaches de l'Ouest (USA). Dans les deux cas, j'analyserai deux modes différents de «faire mémoire »: le récit, qui peut renvoyer à la mythologie, et l'image complexe, liée à l'action rituelle.

\section{AUTEUR}

\section{CARLO SEVERI}

EHESS 